\title{
Information Linkage between Carbon and Energy Markets: Multiplex Recurrence Network Approach
}

\author{
Hua Xu, ${ }^{1,2}$ Minggang Wang $\mathbb{D}^{1,3}$ and Weiguo Yang ${ }^{2}$ \\ ${ }^{1}$ Taizhou College, Nanjing Normal University, Taizhou 225300, Jiangsu, China \\ ${ }^{2}$ Faculty of Science, Jiangsu University, Zhenjiang 212013, Jiangsu, China \\ ${ }^{3}$ School of Mathematical Science, Nanjing Normal University, Nanjing 210042, Jiangsu, China
}

Correspondence should be addressed to Minggang Wang; magic821204@sina.com

Received 26 April 2020; Revised 6 July 2020; Accepted 13 July 2020; Published 11 August 2020

Academic Editor: Dehua Shen

Copyright $(92020$ Hua Xu et al. This is an open access article distributed under the Creative Commons Attribution License, which permits unrestricted use, distribution, and reproduction in any medium, provided the original work is properly cited.

\begin{abstract}
In this paper, a multilayer recurrence network is introduced to examine the information linkage between carbon and energy markets. We first construct a multilayer recurrence network of energy and carbon markets, and we define the information linkage coefficient to measure the linkage relationship between the network layers based on the network microstructure. To measure the mutual leading relationship between carbon and energy markets, we construct a time-delay multilayer recurrence network and introduce the time-delay information linkage coefficient to measure the intersystem interaction. The carbon and energy prices, including West Texas Intermediate crude oil, coal, natural gas, and gasoline, from February 22, 2011, to April 1, 2019, are selected as sample data for empirical analysis. The results show that the linkage relationship between oil, coal, natural gas, and carbon prices presents a U-shaped trend in the second, transitional, and third phases of the European Union carbon market, while the linkage trend of gasoline and carbon prices continues to rise. The mutual leading relationship between energy and carbon prices changes in different stages, and carbon price plays a leading role at the present stage.
\end{abstract}

\section{Introduction}

Currently, climate change and carbon dioxide emission reduction have caused great concern globally. How to cut greenhouse gas emissions down and curb the worldwide warming trend effectively has become a common challenge facing all countries. The carbon trading market is an effective policy tool to control and cut greenhouse gas emissions down, the core of which is to form an effective carbon trading price through the supply and demand mechanism to guide enterprises to make emission reduction decisions. Since February 16, 2005, the international carbon market has shown a rapid growth trend and plays a more and more significant role in curbing global carbon emissions effectively.

With the carbon price playing a more and more significant role in the carbon emission trading system, scholars have used qualitative and quantitative methods to study the factors influencing carbon price fluctuations. A great deal of theoretical and empirical research conducted globally has revealed complex interactions between energy and carbon prices [1-11]. The carbon price has an impact on energy price fluctuations [5]. Meanwhile, energy price also possesses an important effect on carbon price fluctuations $[1,2,7]$, and the interaction between the two markets changes with time $[10,11]$. Existing quantitative researches on the interaction between energy and carbon markets mostly use econometric models, like the cointegrated vector autoregressive (CVAR) model [3], Markov-switching VAR model [4], quantile regression method [9], and threshold DCC-GARCH model [10]. However, there are obvious shortcomings in measuring carbon and energy prices based on econometric models. Since both carbon and energy prices have nonlinear, self-similar, complex, dynamic, and multifractal characteristics, these characteristics lead to a complex nonlinear relationship between them. The modeling assumption of the existing econometrics model requires that the time series is stationary, and the econometrics 
model system is mainly based on the linear relationship of the measurement system, which has poor processing ability for nonlinear and nonstationary data. When using econometric models for empirical analysis, the difference in sampling data often leads to different research conclusions.

For the past few years, the use of network structure to study the correlation between complex systems has received extensive scholarly attention globally. The method's primary thought is to express the complex system using complex network and then reveal the essential characteristics of the system by using the network topology. In this field, a series of algorithms have been developed to convert the nonlinear time series of a single variable into a complex network, such as visibility methods, including natural visibility graph [12], horizontal visibility graph [13], parametric natural visibility graph [14], limited penetrable horizontal visibility graph [15], and parametric modified limited penetrable visibility graph [16], the mapping algorithm for pseudoperiodic time series [17], phase space roughening algorithm [18], and algorithms based on phase space reconstruction $[19,20]$. For multivariate time series, scholars have carried out a significant amount of research on how to analyze multivariate time series using complex networks. Based on existing research, there are two principal approaches of transforming multivariate series into complex networks. The first method is to make each time series a node, calculate the correlation between the time sequences of each node, according to the calculated correlation to determine whether to establish the edge between nodes and, according to this method, build the complex network as a single layer of the network [21, 22]. In the second method, each series is converted into a complex network, which is regarded as a layer of a multilayer network, and the edges between layers are established according to the time relationship, so that multielement time series can be transformed into a multilayer network [23, 24]. At present, using complex network theory to reveal nonlinear systems' characteristics has been widely used in many fields $[25,26]$. However, the current application is only limited to measuring the characteristics of nonlinear systems by using existing network topology indicators, such as measuring the correlation of nonlinear systems by using network degree and clustering coefficient. However, due to the different backgrounds of real nonlinear systems, the reliability of such measurements is relatively low. Therefore, it is worth studying deeply whether we can eliminate the existing network topology index framework and design new indexes to measure the correlation of nonlinear systems from the microperspective of a network. Furthermore, there is a lack of research on how to use complex networks to measure the mutual leading relationship between nonlinear systems, and how to design new indicators based on network microstructure to measure the mutual leading relationship between nonlinear systems has important research value.

To overcome the defects of existing research methods, in terms of theoretical methods, a new index is designed to measure the information linkage among nonlinear systems from the microperspective of a network, and a multilayer complex network with time delay is put forward in this paper first, to the best of our knowledge, and the index with time delay is defined to measure the leading relationship among nonlinear systems. The data of carbon and energy price markets are used for empirical analysis. In the empirical analysis section, the multiplex recurrence network is used to study the information linkage relationship between energy and carbon markets. We construct a carbon-energy multilayer recurrence network and define the information linkage coefficient to measure the linkage relationship between the network layers based on the network microstructure. To measure the mutual guidance relationship between systems, we construct a time-delay multilayer recurrence network, introduce the time-delay information linkage coefficient to measure the intersystem interaction, and define the index to measure the intersystem interaction based on the time-delay information linkage coefficient. We selected the carbon and five energy prices (including those of WTI crude oil, coal, natural gas, and gasoline) from February 22, 2011, to April 1, 2019, as sample data for empirical analysis. Compared with previous studies, the innovative contributions are the following: (1) We construct the carbon market-energy market multilayer recurrence network and time-delay recurrence network for, to the best of our knowledge, the first time; (2) based on the network micro8topological structure, we introduce a new measurement index to measure the information linkage and mutual leading relationship between systems; and (3) we empirically study the linkage and leading relationship between energy and carbon markets in different stages of the European Union (EU) carbon market from the perspective of a recurrence network.

The rest of this paper is organized as follows. We introduce the research methods used in this paper in Section 2. In Section 3, we give the data sources and statistical characteristics of the selected sample data. In Section 4, we study the linkage and mutual leading relationship between energy and carbon markets that are based on the multilayer recurrence network approach. The paper is concluded by Section 5.

\section{Methodology}

2.1. Recurrence Plot and Recurrence Network. Letting $\left\{z_{i}\right\}_{i=1}^{N}$ be a time series, we introduce the embedding dimension $m$ and the embedding delay $\tau$, and then $\left\{z_{i}\right\}_{i=1}^{N}$ can be reconstructed as a trajectory in its phase space with timedelay embedding,

$$
\overrightarrow{y_{i}}=\left(z_{i}, z_{i+\tau}, \ldots, z_{i+(m-1) \tau}\right)
$$

Two state vectors of the reconstructed time series are considered to be recurrent if the second vector falls into an $\varepsilon$ - radius sphere of the first vector. The recurrence matrix $\mathbf{R}$ of the recurrence plot (RP) is defined as follows:

$$
\mathbf{R}_{i, j}=\Theta\left(\varepsilon-\left\|\overrightarrow{y_{i}}-\overrightarrow{t y_{i}}\right\|\right), \quad i, j=1,2, \ldots, M,
$$

where $M$ is the trajectory length, $\Theta(\cdot)$ the Heaviside function (i.e., $\Theta(x)=0$ if $x<0$, and $\Theta(x)=1$ otherwise], and $\|\cdot\|$ a $L_{\infty}$ norm. The simplest measure of recurrence quantification analysis (RQA) is the recurrence rate (RR), 


$$
\mathrm{RR}=\frac{1}{M^{2}} \sum_{i, j=1}^{M} R_{i, j} .
$$

The next is DET, which is the proportion of recurrence dots forming diagonal constitutions (of at least length $s_{\min }$ ) to all recurrence dots,

$$
\mathrm{DET}=\frac{\sum_{s=s_{\min }}^{M} s P(s)}{\sum_{s=1}^{M} s P(s)},
$$

where $P(s)=\sum_{i, j=1}^{M}\left[1-\mathbf{R}_{i-1, j-1}\right]\left[1-\mathbf{R}_{i+s, j+s}\right] \prod_{p=0}^{s-1} \mathbf{R}_{i+p, j+p}$ is the diagonal lines' distribution of length $s$. Then, the diagonal line's average length is as follows:

$$
L=\frac{\sum_{s=s_{\min }}^{M} s P(\varepsilon, s)}{\sum_{s=s_{\min }}^{M} P(\varepsilon, s)},
$$

which is the average time that the two phase points in the trajectory approach each other, which can be used to measure the average predictable time of a system. The measured entropy can be used to find the occurrence diagonal lines the lengths of which are exactly $s$,

$$
\mathrm{ENTR}=\sum_{s=s_{\min }}^{M} p(\varepsilon, s) \ln (\varepsilon, s)
$$

which measures the complexity of the system in terms of the probability of occurrence of diagonal lengths in the RP.

Based on the RP, for the trajectory $\overrightarrow{y_{j}}, i=1,2, \ldots, M$ and $\overrightarrow{y_{i}} \in \mathbb{R}^{m}$, the adjacency matrix of the recurrence network (RN) is defined as follows:

$$
A_{i, j}(\varepsilon)=\Theta\left(\varepsilon-\left\|\overrightarrow{y_{i}}-t \overrightarrow{y_{j}}\right\|\right)-\delta_{i, j}, \quad i, j=1,2, \ldots, M,
$$

where $\varepsilon$ is a threshold value and $\delta_{i, j}$ the Kronecker delta $\left(\delta_{i, j}=1\right.$ if $i=j$; otherwise, $\left.\delta_{i, j}=0\right)$.

2.2. Multiplex Recurrence Network. For univariate time series, we can construct corresponding RPs and RNs, and analyze the time series characteristics according to the RQA analysis approach and the topological structure of the network. For multivariate time series, we must introduce a multilayer network and extract the new information from the primitive multivariate series in graph-theoretical terms to feed automatic classifiers in an elementary, exact, and computationally time-saving process. The steps of mapping an $m$-dimensional multivariate series into a multiplex $\mathrm{RN}$ are as follows: (1) mapping $m$-dimensional multivariate time series into $m$ different RNs that are made up of the equal number of nodes; (2) labeling each node of a RN in accordance with its associated time; and (3) putting the $\alpha$-th $\mathrm{RN}$ derived from the $\alpha$-th time series on the $\alpha$ th layer, and then the multiplex recurrence network is constructed.

We consider an $m$-dimensional multivariate series as follows:

$$
\{\mathbf{x}(t)\}_{t=1}^{N}, \quad \mathbf{x}(t)=\left(x^{1}(t), x^{2}(t), \cdots, x^{M}(t)\right) \in \mathbb{R}^{M} .
$$

Following the steps mentioned above, the RN of the $\alpha$-th component of $\mathbf{x}(t)$ is obtained and located into the associated layer $\alpha$ of the multiplex network. For an instance with $M=3$ time series, these steps are illustrated in Figure 1 . First, we have three time series and construct a phase space for each signal. The RN of the $\alpha$-th component of the time series is calculated by equations (1) and (2) and then placed into layer $\alpha$. Then, the $M$-layer multiplex RN (MRN) is defined,

$$
\mathbf{A}=\left\{A^{[1]}, A^{[2]}, \ldots, A^{[M]}\right\},
$$

where $A^{[\alpha]}=\left\{a_{i j}^{[\alpha]}\right\}, \alpha=1,2, \ldots, M$ and $a_{i j}^{[\alpha]}=1$ if and only if nodes $i$ and $j$ are connected by a link at layer $\alpha$ [24]. Then, the entire multiplex network can be formed according to the supra-adjacency matrix,

$$
\mathbf{A}=\left[\begin{array}{cccc}
A^{[1]} & I_{N} & \cdots & I_{N} \\
I_{N} & A^{[2]} & \ddots & \vdots \\
\vdots & \ddots & \ddots & I_{N} \\
I_{N} & \cdots & I_{N} & A^{[M]}
\end{array}\right]
$$

where $I_{N}$ is the $N$-dimensional identity matrix.

From Figure 1, the linkage relationship between the systems can be represented by the relationship between the network structures of the MRN. Therefore, based on the network connection relationship, we define the information linkage coefficient as follows:

$$
\omega=\frac{\sum_{i} \sum_{j \neq i} \sum_{\alpha} a_{i j}^{[\alpha]}-\sum_{i} \sum_{j \neq i}\left(1-\delta_{\left(0, \sum_{\alpha} a_{i j}^{[\alpha]}\right)}\right)}{(M-1) \sum_{i} \sum_{j \neq i}\left(1-\delta_{\left(0, \sum_{\alpha} a_{i j}^{[\alpha]}\right)}\right)} .
$$

which calculates the information linkage relation between layers according to the connection relation between nodes of each layer. Different from the index defined in previous literature $[22,24], \omega$ takes values in $[0,1]$. For example, $\omega=0$ if there exists a layer $\alpha$ such that $a_{i j}^{[\alpha]}=1$ and $a_{i j}^{[\beta]}=0, \forall \beta \neq \alpha$ (the left-hand panel of Figure 2), while $\omega=1$ only if the network structure is the same at each layer (the right-hand panel of Figure 2). Using equation (11), we can calculate the linkage coefficient of the MRN shown in the middle panel of Figure 2 as 0.3 . In short, $\omega=0$ means that there is no information linkage effect between systems; there exists an information linkage effect when $\omega$ is not equal to zero, and the closer the value of $\omega$ is to 1 , the higher the information linkage effect between systems.

2.3. Time-Delay MRN. To reveal the probability of the occurrence of similar states in different systems with a certain delay $\tau$, we introduce the time-delay MRN (DMRN). For an example with $M=3$ time series, these steps are illustrated in Figure 3. In particular, when $\tau=0$, DMRN degenerates into MRN.

The delay mean edge overlap $\omega_{\tau}$ is introduced to measure the similarity between layers $\alpha$ and $\beta$ with a certain delay $\tau$, 


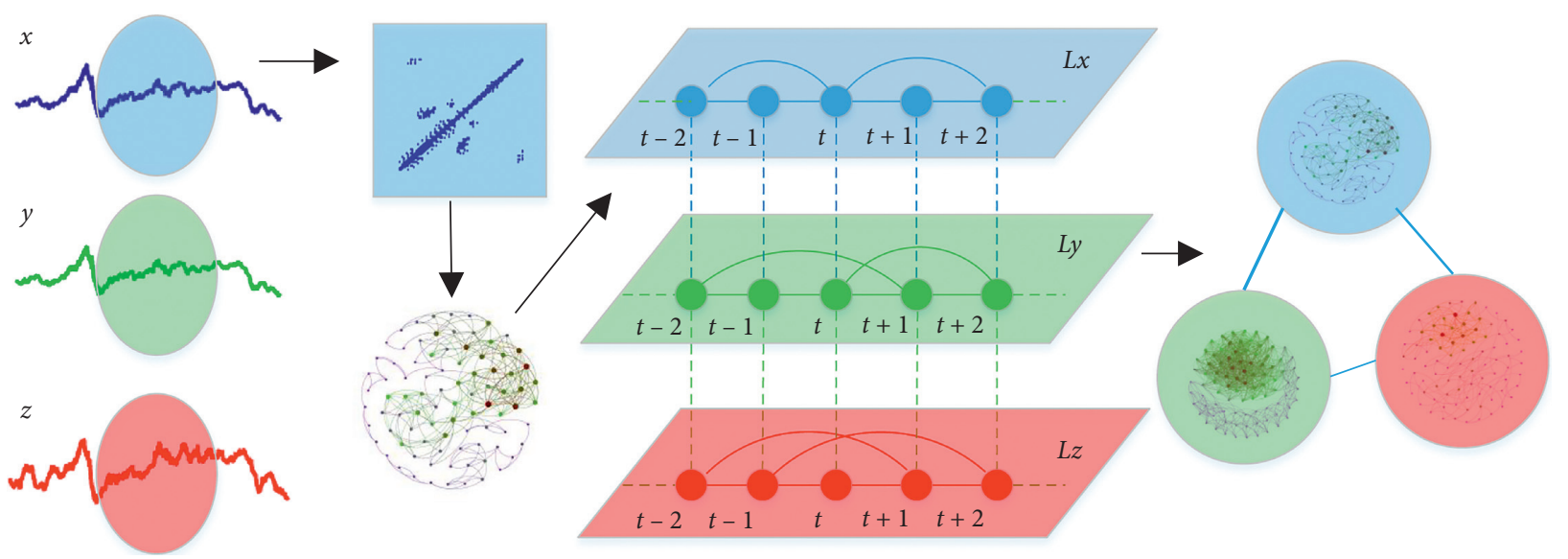

FIGURE 1: The multiplex recurrence network (MRN) algorithm maps a three-dimensional series into a three-layer multiplex recurrence network.
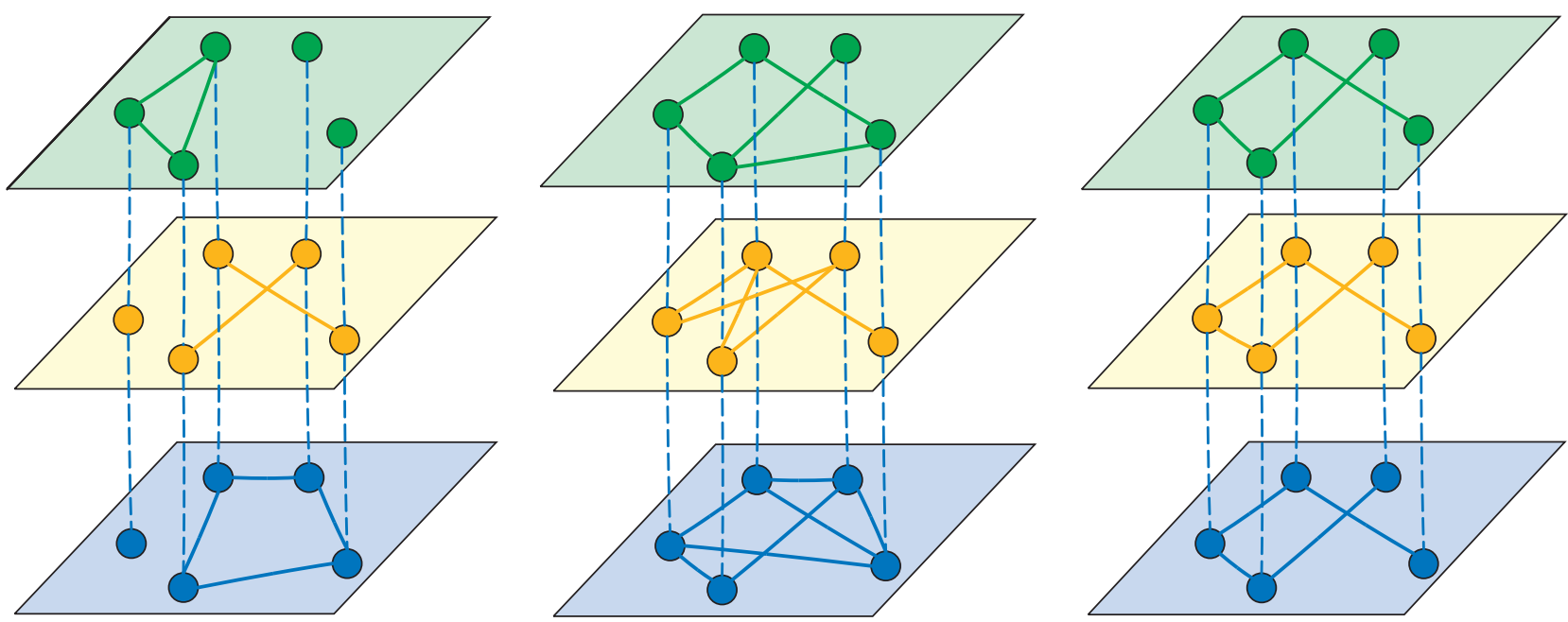

FiguRE 2: Linkage coefficients corresponding to different network structures.
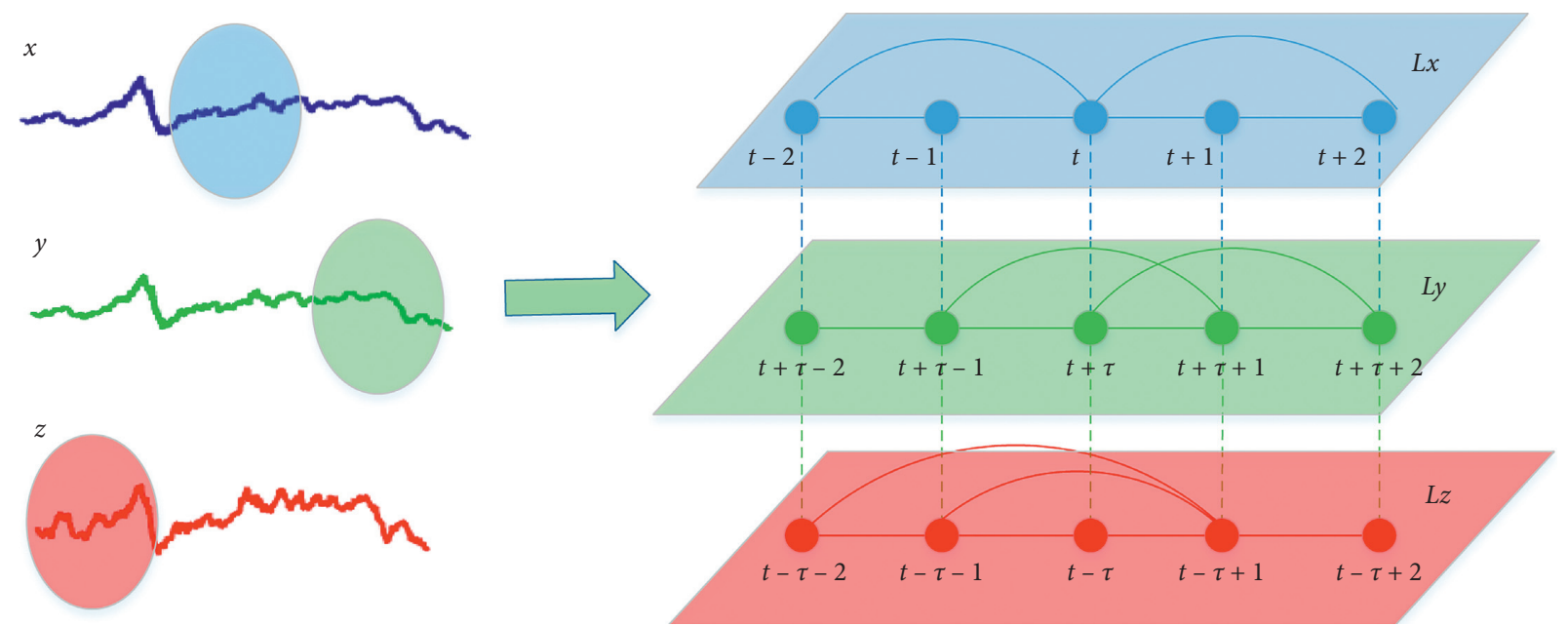

FIGURE 3: The time-delay multiplex recurrence network (DMRN) algorithm maps a three-dimensional time series into a three-layer timedelay MRN. 


$$
\omega_{\tau}=\frac{\sum_{i} \sum_{j \neq i}\left(a_{i j}^{[\alpha, t]}+a_{i j}^{[\beta, t+\tau]}\right)-\sum_{i} \sum_{j \neq i}\left(1-\delta_{\left.0,\left(a_{i j}^{[\alpha, t]}+a_{i j}^{[\beta, t+\tau]}\right)\right)}\right.}{\sum_{i} \sum_{j \neq i}\left(1-\delta_{0,\left(a_{i j}^{[\alpha, t]}+a_{i j}^{[\beta, t+\tau]}\right)}\right)} .
$$

Note that $\omega_{\tau}$ also takes values in $[0,1]$. The higher the value of $\omega_{\tau}$, the higher the linkage relationship between the systems at times $t$ and $t+\tau$.

Next, we define the symmetrical and antisymmetrical combinations as follows:

$$
\begin{aligned}
& Q_{\tau}=\frac{\omega_{\tau}+\omega_{-\tau}}{2}, \\
& q_{\tau}=\frac{\omega_{\tau}-\omega_{-\tau}}{2},
\end{aligned}
$$

which measures the interrelations between two time series and their delay behavior, respectively. Note that $0 \leq Q_{\tau} \leq 1$ and $-1 \leq q_{\tau} \leq 1$. We have $Q_{\tau}=1$ if and only if the two time series are fully synchronized. In addition, if the time series $x$ always precedes $y$, then $q_{\tau}=1$. Thus, we can use $Q_{\tau}$ and $q_{\tau}$ to simply quantify the linkage relationship between two systems and are able to determine which system leads the other.

\section{Data Descriptions}

The day-to-day futures of EUA as carbon prices were selected as sample data. As for energy price, we took WTI crude oil, London gasoline, coal, and natural gas prices derived from the European Energy Exchange. The sample period ranged from February 22, 2011, to April 1, 2019. Considering the comparability of the selected sample data, we only picked the dates out while both sample prices own records. Finally, we obtained 2,029 observations for each price. The EU carbon emission trading system is planned to be developed in a phased and step-by-step manner, which is divided into three stages: first (2005-2007), second (2008-2012), and third (2013-2020) stages. The second and third stages' carbon price data are also included by the sample data that are selected in this paper. Table 1 displays the descriptive statistics of the selected five sample prices.

As is visible from Table 1 , the coefficient of variation (0.5635) of the carbon price data is greater than the energy price, indicating that the carbon price data in the selected sample data have the greatest degree of variation. The skewness of carbon and energy price data is not equal to 0 , in which carbon, WTI crude oil, and natural gas price data are subject to right-skewed distribution, and their skewnesses are 1.2394, 0.0175, and 0.4350 , respectively. Coal and gasoline price data are subject to left-skewed distribution, and their corresponding skewnesses are -0.2138 and -0.0 .0844 , respectively. The kurtosis of carbon price data is greater than 3 , while the kurtosis of energy price data is less than 3 , indicating that the distribution density curve of carbon price data is steeper near its peak than the normal distribution, while the distribution density curve of energy price sample data is gentler near its peak than the normal distribution.
The results of JarqueBera tests show that both carbon and energy price sample data do not obey the normal distribution. The above statistical results show that both carbon and energy prices here all follow the fat-tailed nonnormal distribution. However, it is difficult to describe the selfsimilarity, multifractal, and other complex characteristics of carbon and energy price markets by traditional descriptive statistics. Therefore, we must analyze them from a new perspective.

In the following, we further analyze the characteristics of carbon and energy price data by using the RQA method. First, using equation (2), we give the recurrence plot of energy and carbon and prices in the second stage of development of the EU carbon trading market (from February 22, 2011, to December 31, 2012), as illustrated in Figure 4.

From Figure 4, recurrence can be observed in both carbon and energy price systems, but the patterns of the RPs are rather different. The RP of the Gaussian white noise consists of many single dots. The distribution of the dots in this RP appears to be fairly erratic. However, both RPs of carbon and energy prices are reflected by short diagonal and vertical structures, which means that Gaussian white noise is an unpredictable system, and carbon and energy prices are the less predictable systems. In the specific areas on the RPs of carbon and energy prices, we can also find the roughly equal vertical distances between the diagonal lines, indicating that there are unstable periodic orbits in carbon and energy price systems. These results show that the carbon and energy price sequences are significantly different from the white Gaussian noise sequences, and they both have chaotic characteristics.

In order to quantitatively depict the chaotic characteristics of carbon price and energy price series, we calculate the RQA index of RPs. Since the carbon price sample data contains data from the second and third stages of the EU carbon market, we calculate the RQA indexes corresponding to the carbon price and energy price in these two stages, respectively. Due to the different sample data volume in these two periods, for the convenience of comparative analysis, we select the same sample size data for calculation in these two stages. Therefore, we select the sample data of the second stage from February 22, 2011, to December 31, 2012, and the third stage from May 25, 2017, to April 1, 2019. The results are shown in Table 2.

As is visible from Table 2, in the second stage of the EU carbon trading market, the RQA measure indicators of carbon price sample data include RR, DET, $L$, and ENTR measures that are all greater than the energy price sample data, indicating that the autocorrelation of the carbon price, average predictable time, and the complexity of the system are all higher than the energy price system in this stage. In the EU carbon market's third stage, all the RQA indexes of carbon price data are smaller than those of the second stage, which is closer to the RQA index value of energy price at this stage, indicating that the similarity between energy and carbon price data fluctuation is higher at this stage. 
TABLE 1: The results of the descriptive statistics of the sample data.

\begin{tabular}{|c|c|c|c|c|c|}
\hline & Carbon & WTI oil & Coal & Nature gas & Gasoline \\
\hline Mean & 9.9878 & 72.6916 & 57.9150 & 3.2340 & 704.4379 \\
\hline Std & 5.6280 & 23.5450 & 11.1410 & 0.7268 & 220.4847 \\
\hline Cvar & 0.5635 & 0.3239 & 0.1924 & 0.2247 & 0.3130 \\
\hline Skewness & 1.2394 & 0.0175 & -0.2138 & 0.4350 & -0.0844 \\
\hline Kurtosis & 3.3602 & 1.4615 & 2.5589 & 2.7921 & 1.4958 \\
\hline JarqueBera & $530.4079^{* * *}$ & $200.2190^{* * *}$ & $31.9025^{* * *}$ & $67.6419^{* * *}$ & $193.7020^{* * *}$ \\
\hline
\end{tabular}

Note: ${ }^{* * *}$ denotes significance at $1 \%$ level.
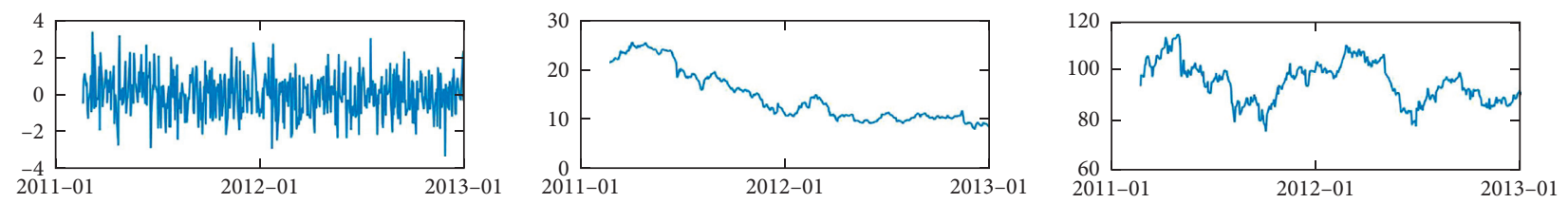

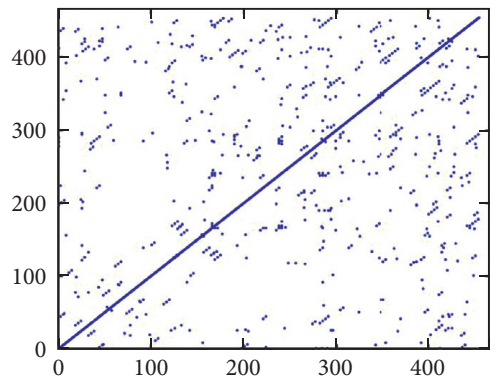

(a)
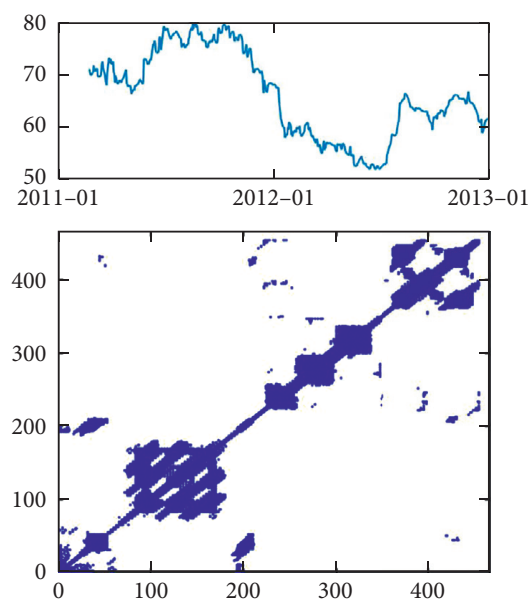

(d)

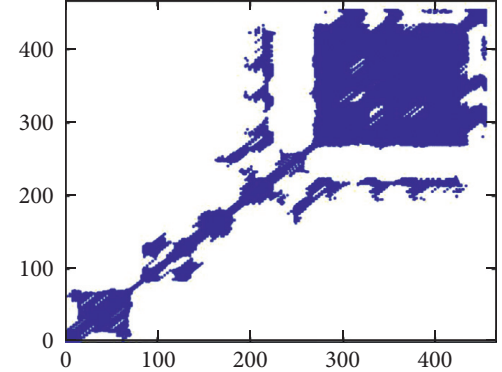

(b)
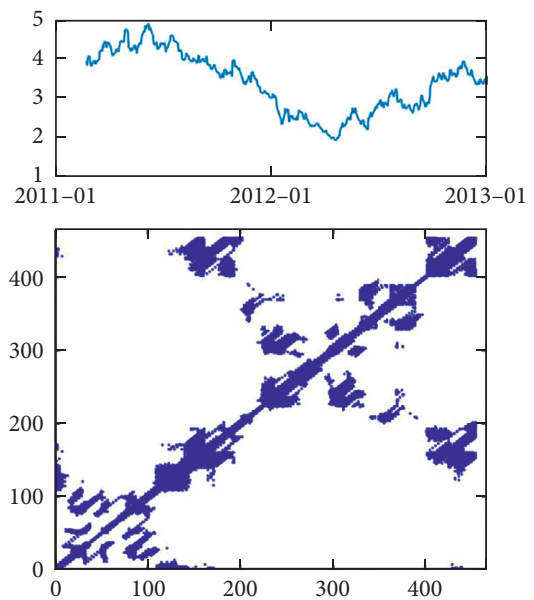

(e)

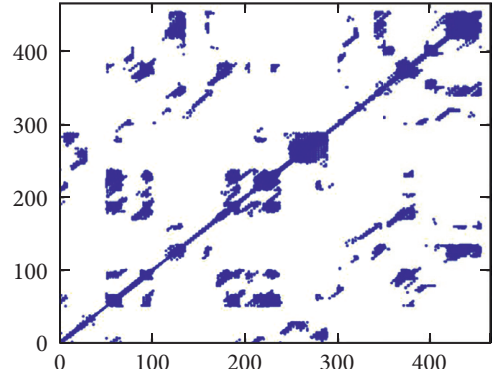

(c)
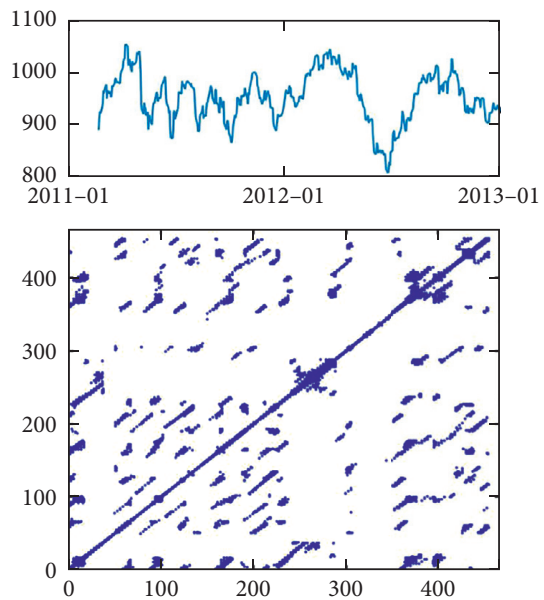

(f)

Figure 4: Recurrence plot of carbon and energy prices from 22 February 2011 to 31 December 2012. (a) Gaussian noise, (b) Carbon price, (c) WTI Crude oil price, (d) Coal price, (e) Natural gas price, (f) Gasoline price.

\section{Information Linkage between Energy and Carbon Markets}

As we know, the EU ETS has finished two phases and stays at the third phase up to now. The sample period in this paper ranged from February 22, 2011, to April 1, 2019. In order to more clearly reveal the dynamic evolution process of the linkage relationship between the carbon market and energy market and maintain the same sample data volume at each stage, the entire sample data period was divided into four periods. The EU ETS's second phase is from February 22,
2011, to December 31, 2012. This phase corresponds to the second phase of the construction and development of the EU carbon market. The transition period from January 27, 2012, to December 5, 2013, is from the second stage to the third stage of the EU ETS. This phase contains the carbon price data of the second and third stages of the development of the EU carbon market. The change of the carbon price data can reveal the influence mechanism of the changes of various policies on the carbon market price during the transition period from the second stage to the third stage. From January 2, 2013, to November 12, 2014, is the beginning of 
TABLE 2: RQA measures of the six series of Gaussian noise, carbon prices, and energy prices.

\begin{tabular}{|c|c|c|c|c|c|c|c|}
\hline & & $\begin{array}{c}\text { Gaussian } \\
\text { noise }\end{array}$ & $\begin{array}{l}\text { Carbon } \\
\text { price }\end{array}$ & Oil price & $\begin{array}{l}\text { Coal } \\
\text { price }\end{array}$ & $\begin{array}{l}\text { Natural gas } \\
\text { price }\end{array}$ & $\begin{array}{l}\text { Gasoline } \\
\text { price }\end{array}$ \\
\hline \multirow{4}{*}{$\begin{array}{l}\text { Second stage of EU ETS (2011/02/22-2012/12/ } \\
\text { 31) }\end{array}$} & $\mathrm{RR}$ & 0.0052 & 0.1985 & 0.0533 & 0.0776 & 0.0731 & 0.0347 \\
\hline & DET & 0.0003 & 0.9649 & 0.7293 & 0.8803 & 0.8328 & 0.6136 \\
\hline & $L$ & 0.0052 & 14.4102 & 3.7267 & 5.8783 & 5.0159 & 2.9973 \\
\hline & ENTR & 0.0091 & 2.2336 & 1.2171 & 1.6204 & 1.4946 & 1.0225 \\
\hline \multirow{4}{*}{$\begin{array}{l}\text { Third stage of EU ETS (2017/05/25-2019/04/ } \\
01)\end{array}$} & $\mathrm{RR}$ & 0.0044 & 0.1177 & 0.0710 & 0.1173 & 0.2552 & 0.0885 \\
\hline & DET & 0.0000 & 0.8685 & 0.8356 & 0.9714 & 0.9124 & 0.8836 \\
\hline & $L$ & 0.0000 & 7.0627 & 4.8162 & 5.5321 & 5.5309 & 5.8236 \\
\hline & ENTR & 0.0000 & 1.4513 & 1.5598 & 2.4583 & 1.8076 & 1.7606 \\
\hline
\end{tabular}

the third phase of the EU ETS. The fluctuations of the carbon price in this stage can be used to assess the short-term effects of the implementation of the new policies in the third stage of the EU carbon market. The current stage of the EU ETS is from November 13, 2014, to April 1, 2019. At this stage, the trend of carbon price fluctuations can be used to evaluate the operation effect of the EU carbon market and predict the future trend of carbon price fluctuations. In each of the four phases, a separate multilayer $\mathrm{RN}$ was built, and equation (11) was used to calculate the linkage coefficient between carbon and energy prices. The results are shown in Figure 5, where the graph in the circle represents the $\mathrm{RN}$ of energy and carbon prices in different phases. From the aspect of RN structure, we can see that the RN with different prices presents different network structures in different periods. Specifically, the RNs corresponding to the four energy prices in different periods have similar network structures. However, the RN structure corresponding to the carbon and energy prices differs significantly in different periods; in the second and transition stages, the RNs corresponding to carbon and energy prices exhibit significant differences (Figures 5(a) and 5(b)). With the gradual maturity of the EU carbon trading market, the network structure of the recursive network of carbon and energy markets shows a high similarity (Figures 5(c) and 5(d)), which is consistent with the conclusions drawn in Section 3. In fact, the topological structure of RNs at different periods can be used to quantitatively study the formation mechanism of carbon market price, which will be elaborated in future papers but will not be discussed in detail here.

From Figure 5(a), in the EU ETS's second stage, the average linkage coefficient between energy prices is 0.5248 , among which WTI crude oil price has the highest correlation with gasoline price, and the linkage coefficient is 0.6508 . The average linkage coefficient between energy and carbon prices is 0.5302 , among which the linkage between natural gas and carbon prices is the strongest. In this stage, the average linkage relationship between energy prices is slightly weaker than that between energy and carbon prices. From Figure 5(b), in the transition period from the second to the third stage of EU ETS, the average linkage coefficient between the energy price is 0.4806 , which has the highest linkage relationship between natural gas and gasoline prices and the linkage coefficient is 0.6268 in this stage, and compared with the second stage the average linkage relationship fell by $8.42 \%$. Especially for the linkage relationships between coal and natural gas prices and between coal and gas prices, the linkage relationship between crude oil and gasoline prices fell by $32.89 \%, 21.23 \%$, and $23.40 \%$, respectively. The average linkage coefficient between energy and carbon prices is 0.4894 . Compared with the second stage, the average linkage coefficient between energy and carbon prices decreased by $7.70 \%$, among which the linkage relationship between crude oil, coal, natural gas, and carbon prices decreased by $4.84 \%, 34.14 \%$, and $1.04 \%$, respectively. At this stage, the average linkage relationship between energy prices is still slightly weaker than that between energy and carbon prices. From Figure 5(c), early in the EU ETS's third stage the average linkage coefficient between the energy price is 0.6150 . WTI crude oil and gasoline prices had the highest linkage relationship, with a linkage coefficient of 0.8028. In this stage, compared with the transition stage, the linkage relationship between energy prices was significantly enhanced, with the average linkage relationship coefficient increased by $21.85 \%$, and the oil-natural gas, crude oilgasoline, coal-natural gas, coal-gasoline, and natural gasgasoline prices rose by $23.36 \%, 37.90 \%, 19.46 \%, 25.68 \%$, and $18.70 \%$, respectively. The average linkage relationship between energy and carbon prices is 0.6092 , and compared with the transition phase, the correlation was significantly enhanced. The average linkage coefficient increased by $21.85 \%$, and all the linkage relationships between WTI oil, coal, natural gas, gasoline, and carbon prices increased significantly, by $26.37 \%, 21.84 \%, 12.63 \%$, and $19.55 \%$, respectively. Unlike the second and transition phases, the average linkage relationship between energy prices in this phase is stronger than that between energy and carbon prices. From Figure 5(d), in the current stage, the average linkage coefficient between energy prices is 0.6814 . WTI crude oil and gasoline prices had the highest linkage relationship, with a linkage coefficient 0.8726 . Compared with the beginning of the third stage, the linkage relationship between the energy prices increased, with the average linkage coefficient increased by $9.74 \%$, and the crude oilcoal, crude oil-natural gas, crude oil-gasoline, coal-natural gas, coal-gasoline, and natural gas-gasoline prices increased by $12.18 \%, 6.28 \%, 8 \%, 17.08 \%, 16.91 \%$, and $0.74 \%$, respectively. In this stage, the average linkage relationship between energy prices is stronger than that between energy and carbon prices.

The above-mentioned results indicate that in different stages of the development of EU carbon market, the linkage relationship between the carbon market and energy market presents different trends, which is mainly determined by the 


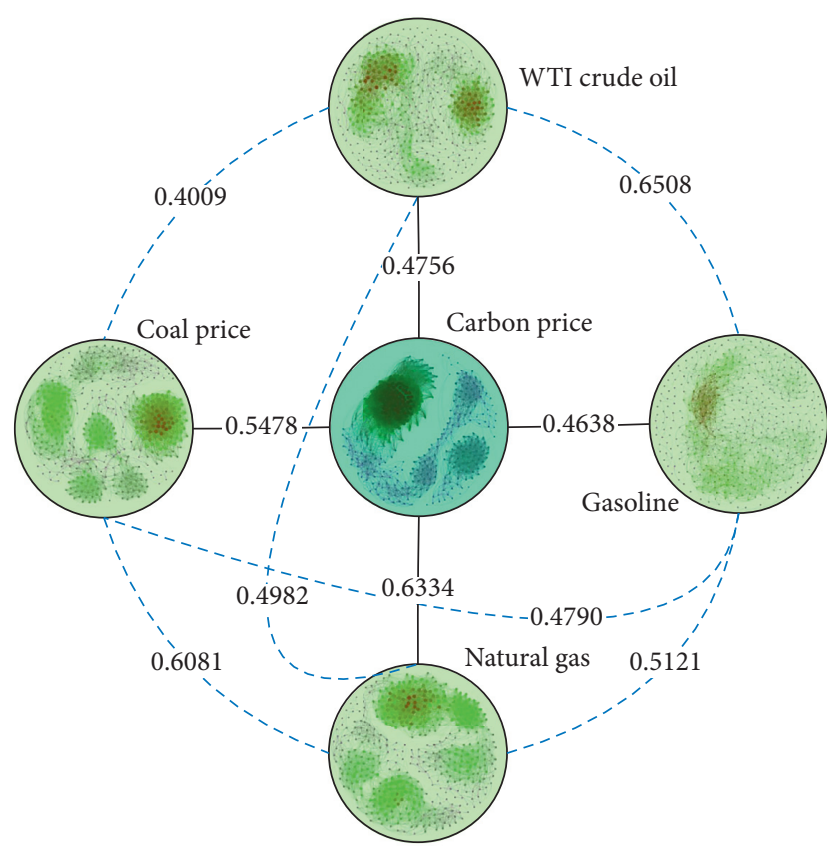

(a)

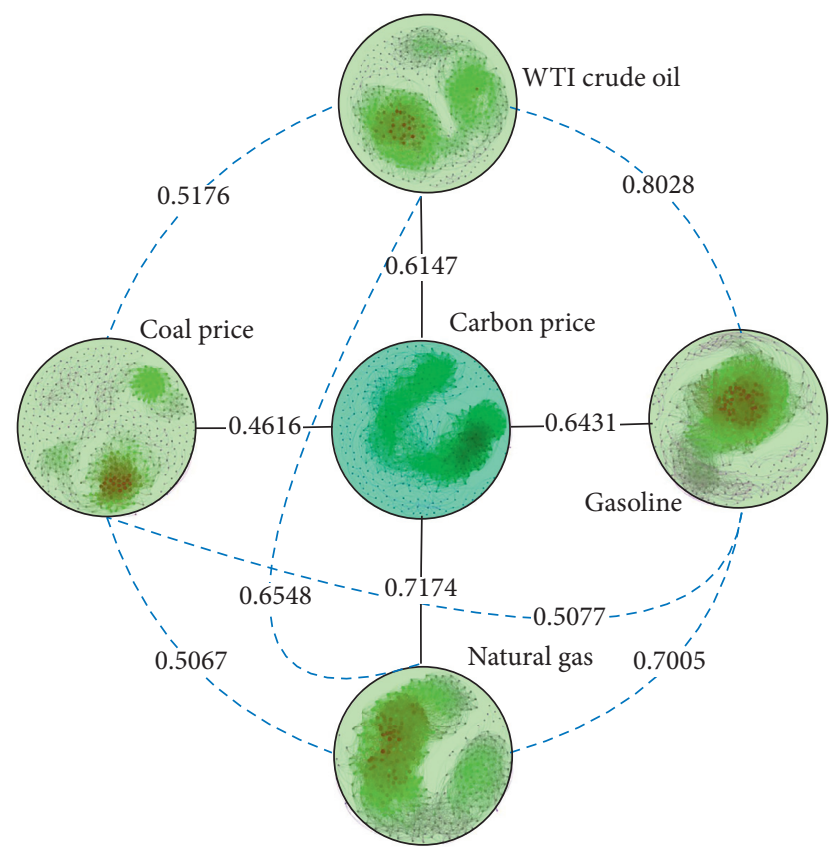

(c)

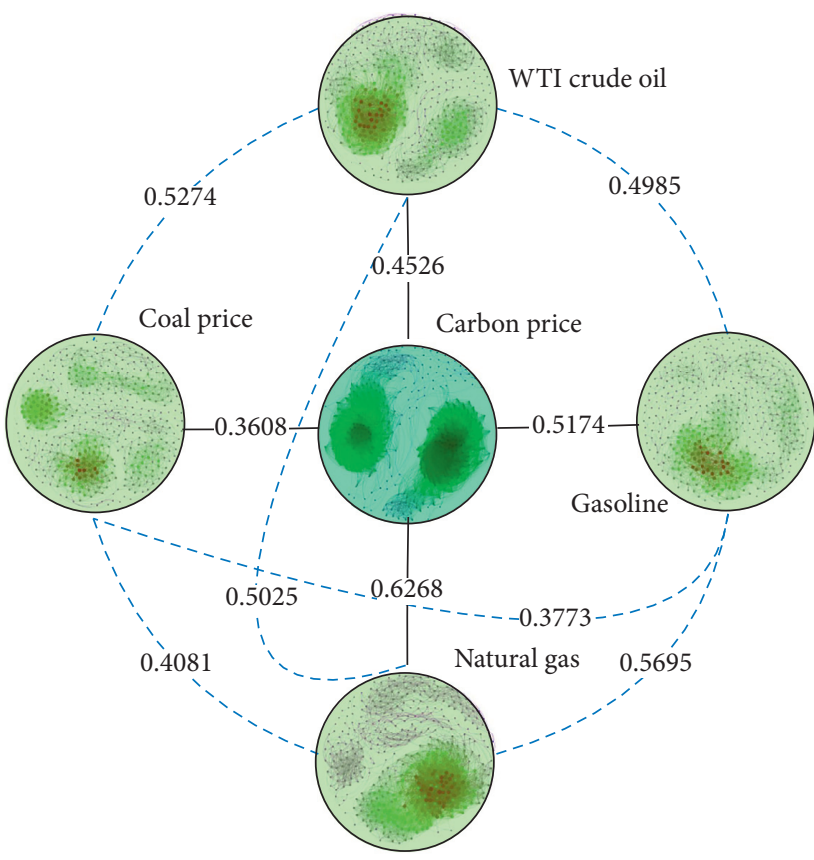

(b)

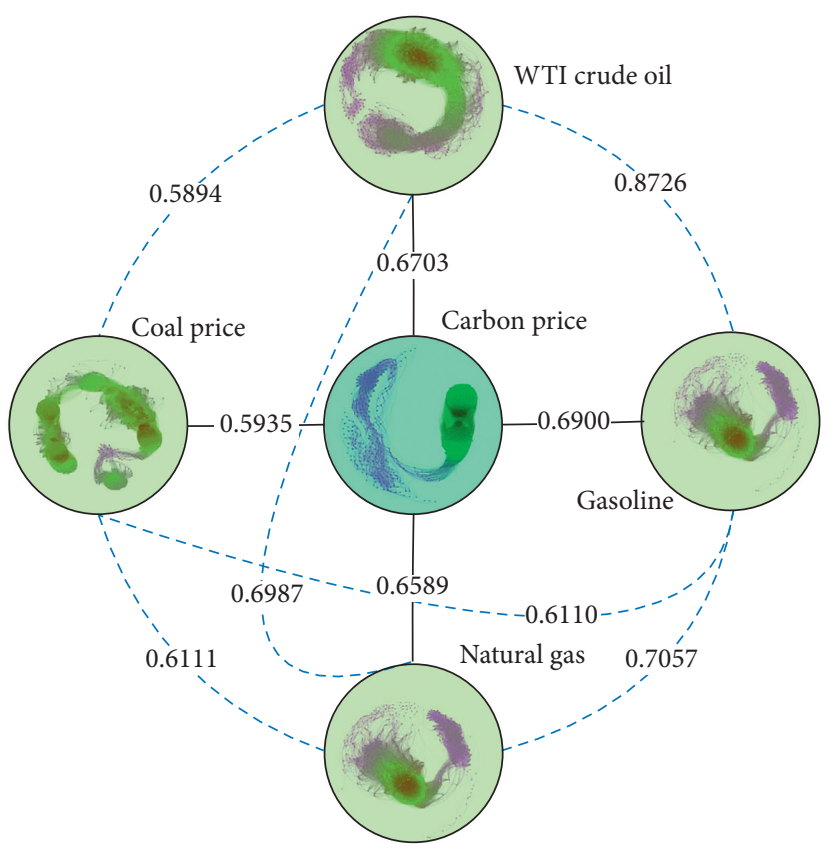

(d)

FiguRe 5: Linkage between carbon and energy prices: (a) second stage, (b) transition from second to third stage, (c) initial stage of third stage, and (d) current stage.

property of the carbon price itself and the maturity degree of the development of EU ETS. First, carbon emission rights are a special commodity produced by effective demand and scarcity, and carbon price is naturally characterized by virtuality, equity, policy, and volatility, which are different from other common commodities. Second, significant differences exist in the EU carbon trading system's working mechanism in the second and third stages. The second stage is EU ETS's development stage, in which the carbon emission rights' initial allocation scheme is primarily in the form of free allocation, while carbon emissions initial allocation is the core of the impact on carbon price, although free allocation is a simple and effective way to ensure the operation of the carbon market, which can promote a reduction ability to buy time for the enterprise, but this approach can hinder market prices and play a signaling role, leading to the distortion of market efficiency and, eventually, to the RQA indexes of carbon and energy prices in this phase 


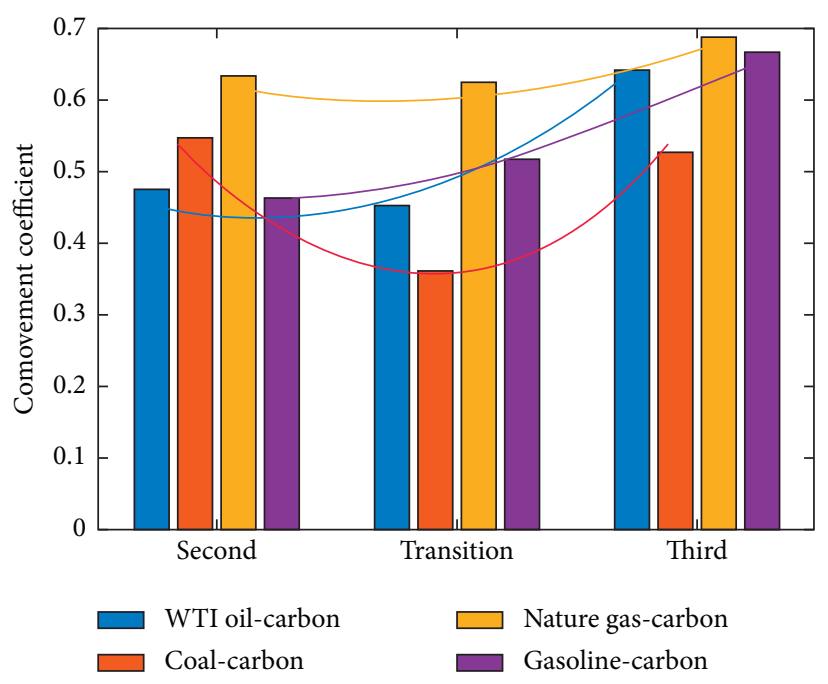

(a)

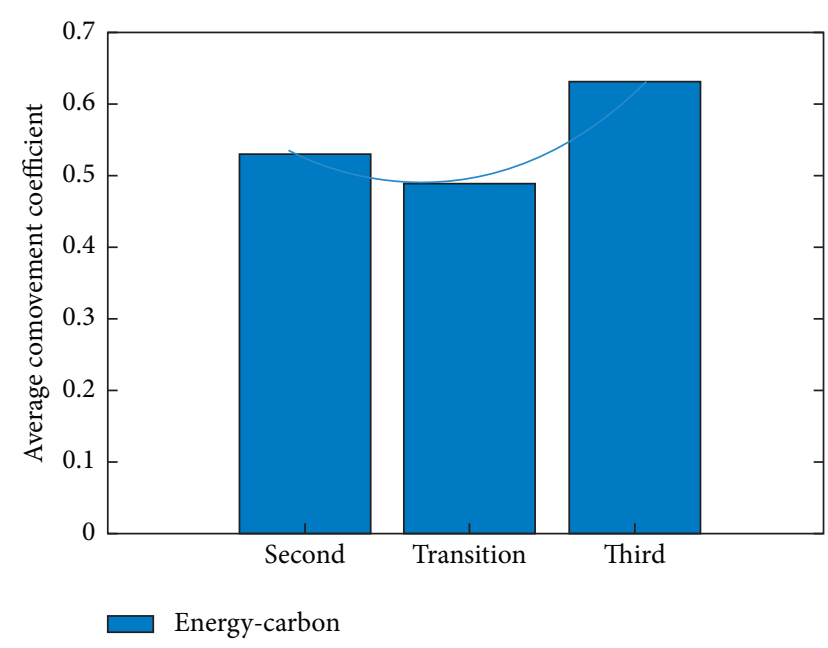

(b)

FiguRe 6: (a) Linkage relationship between crude oil, coal, natural gas, gasoline, and carbon prices in different stages. (b) Average linkage between energy and carbon prices.

to exhibit a significant difference. The third stage is the mature reduction development stage of EU ETS. In this phase, the EU on the trading main body adopted an innovative baseline distribution method, and the original free allocation gradually transitioned to an auction. These measures led to carbon price really playing a role as a market signal, and the carbon and energy markets exhibited more similar market attributes. Therefore, there is a higher similarity between the volatility complexity of carbon and energy prices.

Based on the above analysis, the linkage connection between crude oil, coal, natural gas, and carbon prices presents a U-shaped trend of first decreasing and then increasing in the second, transition, and third stages of the EU carbon market, while the linkage trend of gasoline and carbon price continues to increase, as shown in Figure 6(a). On the whole, energy and carbon prices show a stronger linkage trend with the gradual maturity of the EU carbon market, as shown in Figure 6(b).

Continuing our discussion of the mutual leading relationship, we use the change of $\tau$ in the interval $[0,60]$ and use equation (12) to calculate the lagging linkage coefficients $\omega_{\tau}$ and $\omega_{-\tau}$. Then, the values of indicators $Q_{\tau}$ and $q_{\tau}$ are calculated using equation (13). The results are illustrated in Figure 7.

Figure 7 shows that in the second stage of the EU ETS the maxima for the symmetry measure $Q_{\tau}$ at a time lag are $\tau=28,1,34$, and 34 for the carbon-oil, carbon-coal, carbon-natural gas and carbon-gasoline systems, respectively, which reveals the synchronization of carbon-oil, carboncoal, carbon-natural gas, and carbon-gasoline after lags of $28,1,34$, and 34 . The values of the asymmetry measure $q_{\tau}$ at the corresponding lag is negative, positive, negative, and positive, respectively, which reveals that coal and gasoline prices lead carbon price, and that carbon price leads crude oil and natural gas prices. In the transition period from the second to the third stage, the maxima for the symmetry measure $Q_{\tau}$ at time lags $\tau=54,13,34$, and 52 for carbonoil, carbon-coal, carbon-natural gas, and carbon-gasoline systems, respectively, reveal the synchronization of carbonoil, carbon-coal, carbon-natural gas, and carbon-gasoline after lags of 54, 13, 34, and 52. The values of the asymmetry measure $q_{\tau}$ at the corresponding lag are positive, positive, negative, and positive, respectively, which reveal that crude oil, coal, and gasoline prices lead carbon price, and carbon price leads to natural gas price. In the beginning of the third stage, the maxima for the symmetry measure $Q_{\tau}$ at time lags of $48,20,3$, and 50 for carbon-oil, carbon-coal, carbonnatural gas, and carbon-gasoline systems, respectively, reveal the synchronization of carbon-oil, carbon-coal, carbon-natural gas, and carbon-gasoline after lags of 48, 20, 3 , and 50 . The values of the asymmetry measure $q_{\tau}$ at the corresponding lags are positive, negative, positive, and positive, respectively, which reveal that oil, natural gas, and gasoline lead carbon price, and that carbon price leads coal price. In the current stage, the maxima for the symmetry measure $Q_{\tau}$ at time lags of $60,56,44$, and 3 for carbon-oil, carbon-coal, carbon-natural gas, and carbon-gasoline systems, respectively, reveal the synchronization of carbonoil, carbon-coal, carbon-natural gas, and carbon-gasoline after lags of $60,56,44$, and 3 . The values of the asymmetry measure $q_{\tau}$ at the corresponding lags are all negative, revealing that carbon price leads to oil, coal, natural gas, and gasoline prices, i.e., carbon price leads all energy prices. These consequences suggest that with the EU carbon market's gradual maturity carbon price plays an more and more significant role in leading energy price fluctuation's tendency.

\section{Discussion and Conclusions}

In this paper, the multilayer and time-delay RNs are constructed for the first time, to the best of our knowledge, to study the information linkage relationship between carbon 


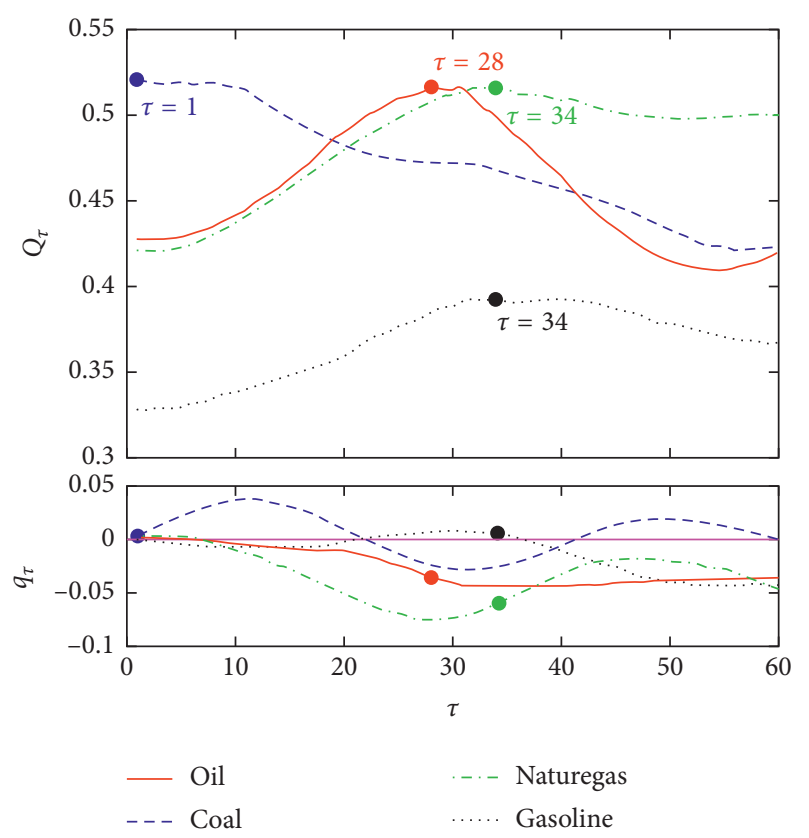

(a)

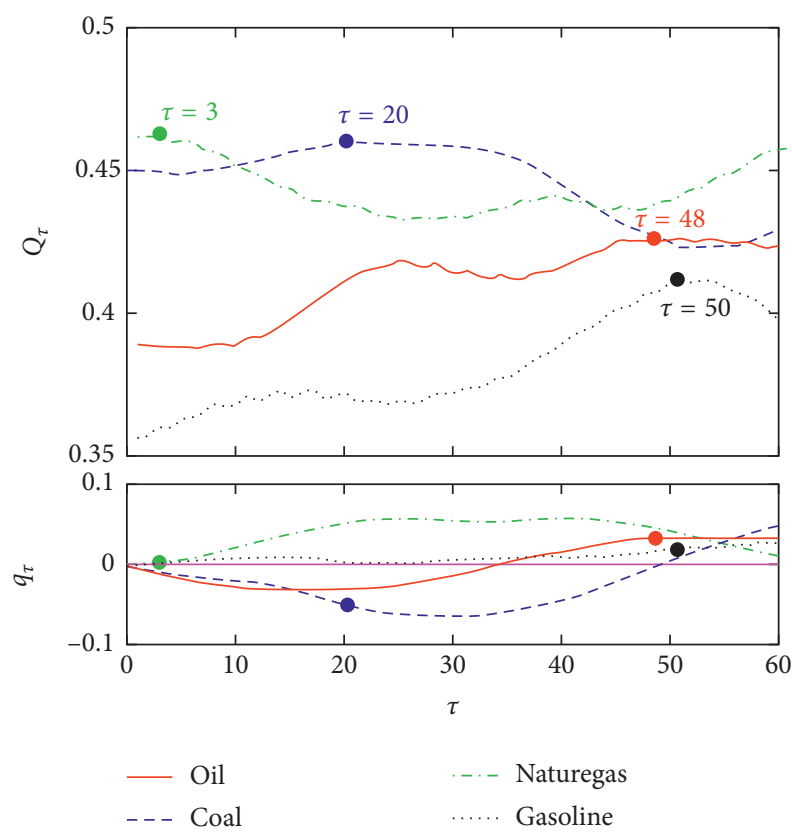

(c)

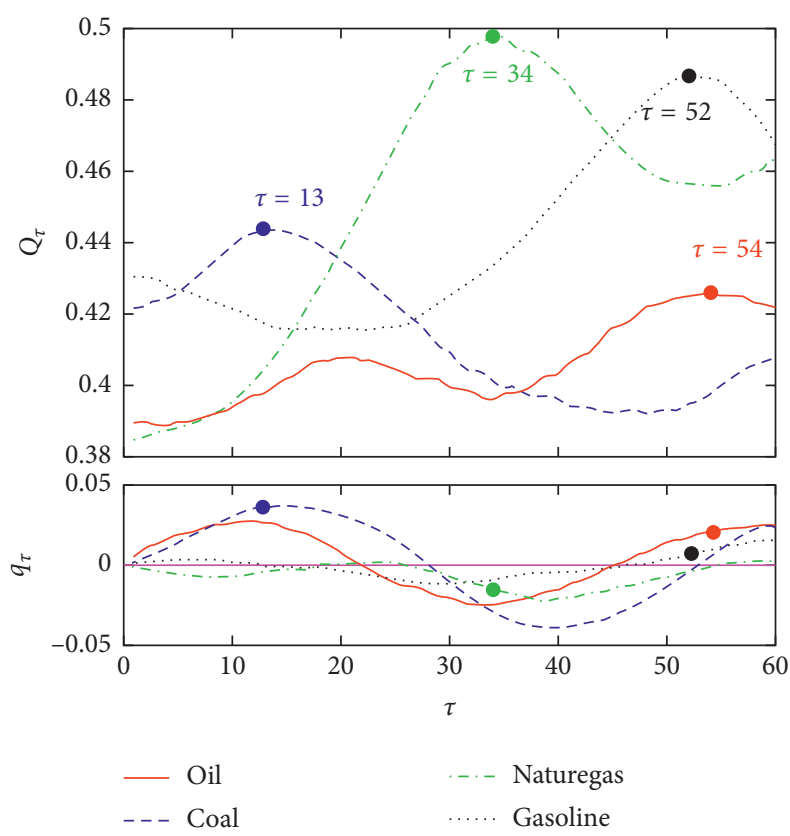

(b)

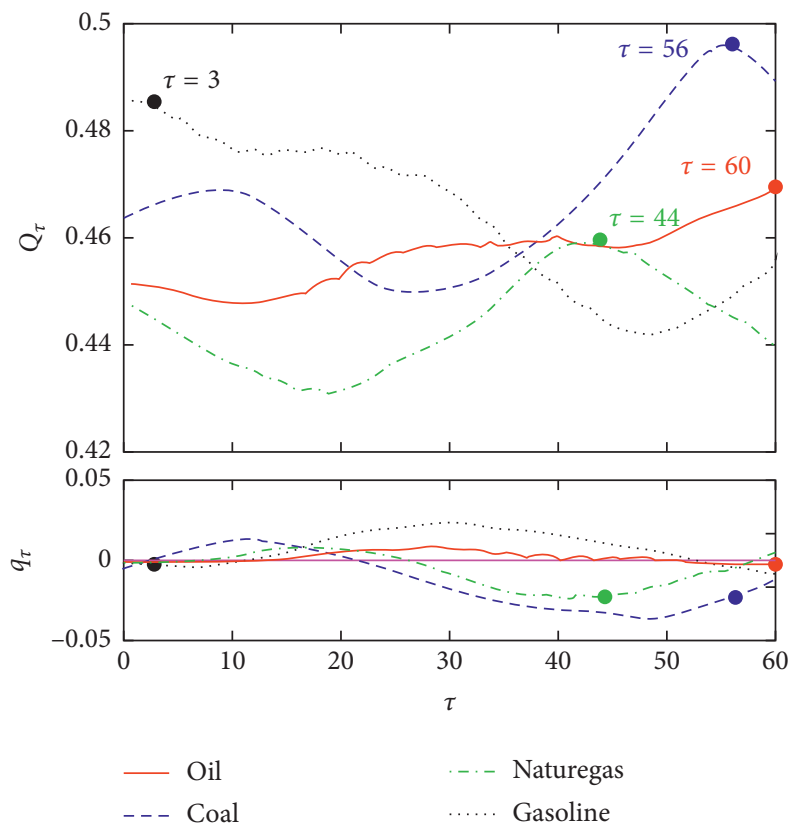

(d)

FIGURE 7: Mutual leading relationship between carbon and energy prices: (a) second stage, (b) transition from second to the third stage, (c) initial stage of the third stage, and (d) current stage.

and energy markets. Based on the microtopological structure of the MRN, we introduce the information linkage coefficient and time-delay information linkage coefficient to measure the linkage relationship and the mutual leading relationship between carbon and energy markets in different stages of the EU carbon market.

In the empirical analysis section, we choose data from February 22, 2011, to April 1, 2019, for carbon and five energy prices as sample data. First, the characteristics of carbon and energy price data were analyzed by using the
RQA method. Then, the entire sample data period was divided into four periods, and within the four stages a multilevel recursive network research relationship was built between carbon and energy prices. It was found that the linkage relationship between crude oil, coal, natural gas, and carbon prices presents a U-shaped trend of first decreasing and then increasing in the second, transitional, and third stages of the EU carbon market, while the linkage trend of gasoline and carbon prices continues to rise. On the whole, energy and carbon prices show a stronger linkage trend with 
the gradual maturity of the EU carbon market. Then, based on the time-delay multilayer recursive network, we studied the mutual leading relationship between energy and carbon markets at different stages of the development of the EU carbon market. It is found that the mutual leading relationship between energy and carbon prices changes in different stages, and carbon price plays a leading role in energy price at the present stage.

Our findings in this paper are helpful for carbon market dealers and energy policy makers. For market traders, it is important to carefully evaluate the dynamic linkages between different periods of time, especially when energy and carbon market conditions are changing, in order to design better hedging strategies. Secondly, comprehending the mutual dominance and price link between energy and carbon markets will permit energy policy makers to lay policies and carbon market mechanisms out to respond to market emergencies more effectively and to ensure the healthy development of both markets. Generally, our study offers a novel systematical viewpoint for comprehending the mutual dominance and information transfer mechanism between energy and carbon markets from the perspective of network micro. It also provides important results for China and other countries to consider in developing emissions trading systems.

The approach that is put forward in this paper can be used to analyze the interaction and mutual guidance among systems in other fields. In the future work, we will further propose new indicators to measure the interaction between systems based on the network structure, so as to enrich and improve the research on the correlation between complex systems based on complex networks.

\section{Data Availability}

Data used to support the findings of this study are available from the corresponding author upon request.

\section{Conflicts of Interest}

The authors declare that they have no conflicts of interest.

\section{Acknowledgments}

The research was supported by the following foundations: the Philosophy and Social Science Research Project in Colleges and Universities of Jiangsu Province (2018SJA2123), the National Natural Science Foundation of China (71503132, 71811520710, 11571142, 91546118), Qing Lan Project of Jiangsu Province (2017), and Six Talent Peaks Project in Jiangsu Province (JY-055).

\section{References}

[1] M. M. Bataller, A. P. Tornero, and E. Valor, "CO2 prices, energy and weather," The Energy Journal, vol. 28, no. 3, pp. 73-92, 2007.

[2] J. H. Keppler and M. Mansanet-Bataller, "Causalities between $\mathrm{CO} 2$, electricity, and other energy variables during phase I and phase II of the EU ETS," Energy Policy, vol. 38, no. 7, pp. 3329-3341, 2010.

[3] H. Fell, "EU-ETS and nordic electricity: a CVAR analysis," The Energy Journal, vol. 31, no. 2, pp. 1-25, 2010.

[4] J. Chevallier, "A model of carbon price interactions with macroeconomic and energy dynamics," Energy Economics, vol. 33, no. 6, pp. 1295-1312, 2011.

[5] D. Kirat and I. Ahamada, "The impact of the European Union emission trading scheme on the electricity-generation sector," Energy Economics, vol. 33, no. 5, pp. 995-1003, 2011.

[6] S. Kumar, S. Managi, and A. Matsuda, "Stock prices of clean energy firms, oil and carbon markets: a vector autoregressive analysis," Energy Economics, vol. 34, no. 1, pp. 215-226, 2012.

[7] P. Aatola, M. Ollikainen, and A. Toppinen, "Price determination in the EU ETS market: theory and econometric analysis with market fundamentals," Energy Economics, vol. 36, pp. 380-395, 2013.

[8] S. J. Byun and H. Cho, "Forecasting carbon futures volatility using GARCH models with energy volatilities," Energy Economics, vol. 40, pp. 207-221, 2013.

[9] S. Hammoudeh, D. K. Nguyen, and R. M. Sousa, "Energy prices and $\mathrm{CO} 2$ emission allowance prices: a quantile regression approach,” Energy Policy, vol. 70, pp. 201-206, 2014.

[10] Y.-J. Zhang and Y.-F. Sun, "The dynamic volatility spillover between European carbon trading market and fossil energy market," Journal of Cleaner Production, vol. 112, pp. 26542663, 2016.

[11] Q. Ji, D. Zhang, and J.-B. Geng, "Information linkage, dynamic spillovers in prices and volatility between the carbon and energy markets," Journal of Cleaner Production, vol. 198, pp. 972-978, 2018.

[12] L. Lacasa, B. Luque, F. Ballesteros, J. Luque, and J. C. Nuno, "From time series to complex networks: the visibility graph," Proceedings of the National Academy of Sciences, vol. 105, no. 13, pp. 4972-4975, 2008.

[13] B. Luque, L. Lacasa, F. Ballesteros, and J. Luque, "Horizontal visibility graphs: exact results for random time series," Physical Review E, vol. 80, no. 4, Article ID 046103, 2009.

[14] I. V. Bezsudnov and A. A. Snarskii, "From the time series to the complex networks: the parametric natural visibility graph," Physica A: Statistical Mechanics and Its Applications, vol. 414, pp. 53-60, 2014.

[15] M. Wang, A. L. M. Vilela, R. Du et al., "Exact results of the limited penetrable horizontal visibility graph associated to random time series and its application," Scientific Reports, vol. 8, no. 1, p. 5130, 2018.

[16] X. Li, M. Sun, C. Gao, D. Huan, and M. Wang, "The parametric modified limited penetrable visibility graph for constructing complex networks from time series," Physica A: Statistical Mechanics and Its Applications, vol. 492, pp. 1097-1106, 2018.

[17] X. Han, J. Zhang, and M. Small, "Superfamily phenomena and motifs of networks induced from time series," Proceedings of the National Academy of Sciences, vol. 105, no. 50, pp. 19601-19605, 2008.

[18] M. Wang and L. Tian, "From time series to complex networks: the phase space coarse graining," Physica A: Statistical Mechanics and Its Applications, vol. 461, pp. 456-468, 2016.

[19] J. Zhang and M. Small, "Complex network from pseudoperiodic time series: topology versus dynamics," Physical Review Letters, vol. 96, no. 23, Article ID 238701, 2006.

[20] R. V. Donner, Y. Zou, J. F. Donges, N. Marvan, and J. Kurths, "Recurrence networks-a novel paradigm for nonlinear time 
series analysis," New Journal of Physics, vol. 12, no. 3, Article ID 033025, 2010.

[21] K. Iwayama, Y. Hirata, K. Takahashi et al., "Characterizing global evolutions of complex systems via intermediate network representations," Scientific Reports, vol. 2, no. 1, p. 423, 2012.

[22] L. Lacasa and J. Iacovacci, "Visibility graphs of random scalar fields and spatial data," Physical Review E, vol. 96, no. 1, Article ID 012318, 2017.

[23] L. Lacasa, V. Nicosia, and V. Latora, "Network structure of multivariate time series," Scientific Reports, vol. 5, no. 1, Article ID 15508, 2015.

[24] D. Eroglu, N. Marwan, M. Stebich, and J. Kurths, "Multiplex recurrence networks," Physical Review E, vol. 97, no. 1, Article ID 012312, 2018.

[25] S. Sannino, S. Stramaglia, L. Lacasa, and D. Marinazzo, "Visibility graphs for fMRI data: multiplex temporal graphs and their modulations across resting-state networks," Network Neuroscience, vol. 1, no. 3, pp. 208-221, 2017.

[26] S. Marinazzo, X. Gao, M. Jiang, and X. Sun, "Multivariate financial time series in the light of complex network analysis," Physica A: Statistical Mechanics and Its Applications, vol. 503, pp. 1241-1255, 2018. 